\title{
Der Intensiv-Fortbildungskurs »Wirtschafts- deutsch für Hochschullehrkräfte in Litauen«. Ein Erfahrungsbericht
}

\author{
Margit Breckle
}

\section{Zusammenfassung}

Es wird eine zweiwöchige Fortbildung vorgestellt, die im Juni 2007 an der Pädagogischen Universität Vilnius (Litauen) stattfand. Der Fortbildungskurs »Wirtschaftsdeutsch für Hochschullehrkräfte in Litauen ", der aus Sicht von Kursorganisator und Gastdozentin wie auch aus Perspektive der Teilnehmerinnen als insgesamt sehr gelungen bezeichnet werden kann, wird in organisatorischer und inhaltlicher Hinsicht präsentiert. Der Erfahrungsbericht möchte auch Anregungen für Fortbildungen dieser Art geben.

\section{Einleitung}

Im Juni 2007 fand an der Pädagogischen Universität Vilnius (Litauen) im Rahmen des DAAD-Programms Deutsch für Lehrkräfte im studienbegleitenden Fremdsprachenunterricht in MOE/GUS ${ }^{1}$ eine zweiwöchige Fortbildung Wirtschaftsdeutsch für Hochschullehrkräfte in Litauen statt. Der Fortbildungskurs wurde vom dortigen Lektor des DAAD Klaus Geyer organisiert; als Gastdozentin fungierte Margit Breckle, ehemalige DAAD-Lektorin an der Schwedischen Wirtschaftsuniversität in Helsinki/Finnland. Sowohl aus Sicht des Kursorganisators und der Gastdozentin als auch aus der Perspektive der Teilnehmerinnen kann die als zweiwöchiger Intensiv-Fortbildungskurs betitelte Veranstaltung als insgesamt sehr gelungen bezeichnet werden.

Im vorliegenden Beitrag, der auch Anregungen für Fortbildungen dieser Art geben möchte, wird der Fortbildungskurs in organisatorischer und inhaltlicher Hinsicht präsentiert.

\section{Konzept und Organisation der Fort-} bildung

\subsection{Zielgruppe, Einladung und Anmel- dung}

Der Kurs richtete sich an Hochschullehrkräfte an Universitäten und Kollegien ${ }^{2}$ in

1 Ziel des Programms ist, die in diesen Kursen tätigen Lehrkräfte - in der Regel DeutschlehrerInnen oder GermanistInnen - durch Fortbildung zu unterstützen. Nähere Auskünfte zu diesem DAAD-Programm erteilt das Referat 324 im DAAD.

2 Kollegien sind tertiäre Bildungseinrichtungen, die teils eher Fachhochschulen, teils eher Berufsfachschulen vergleichbar sind. 
Litauen, die an ihren Institutionen Wirtschaftsdeutsch unterrichten.

Die Einladung samt Anmeldeformular für den Kurs wurde in der recht übersichtlichen litauischen Hochschullandschaft an die in Frage kommenden Institute der Universitäten und Kollegien direkt per E-Mail verschickt; zudem wurden bestehende Kontakte zu potenziell Interessierten und MultiplikatorInnen genutzt. Auf den Internetseiten des Kursorganisators wurde zudem eine eigene Seite ${ }^{1}$ für den Kurs eingerichtet.

Die Teilnehmerzahl war von vornherein auf 20 begrenzt. Bei der Bewerbung war eine kurze Begründung vorgesehen, um ggf. eine begründete Entscheidung über die Vergabe der Kursplätze treffen zu können.

\subsection{Kursziel}

Ziel des Kurses war die Fortbildung litauischer Hochschullehrkräfte für Wirtschaftsdeutsch in wirtschaftsfachlicher, fachsprachlicher und didaktischer Hinsicht.

Der Begriff Wirtschaftsdeutsch umfasst dabei einen recht unübersichtlichen Bereich:

1. Im Sinne von fach- oder wissenschaftssprachlichem Deutsch eine theoretische Metaebene der Wirtschaftswissenschaften (vgl. Hundt 1998)

2. DaF-disziplinentypisch die unterrichtliche Vorbereitung »auf außer unterrichtliche Kommunikation" (Reuter 2001: 579). Dabei lassen sich zum einen die studienvorbereitende und -begleitende Vermittlung von Wirtschaftsdeutsch (vgl. z.B. Buhlmann/Fearns 1989; 1995) und zum anderen der berufsbezogene Wirtschaftsdeutsch-Unterricht unterscheiden, d.h. ange- wandtes Deutsch für die Geschäftsbzw. Unternehmenskommunikation (vgl. z. B. Bolten 1993).

Gegenstand der Fortbildung war der berufsbezogene Wirtschaftsdeutsch-Unterricht. Im Mittelpunkt standen dabei typische mündliche und schriftliche Textsorten der Geschäfts- bzw. Unternehmenskommunikation auf Deutsch, deren Vermittlung sowie die Konzeption und Erstellung von Lehr- und Lernmaterialien zur Vermittlung dieser Textsorten. Darüber hinaus sollte der Kurs den Teilnehmerinnen die Möglichkeit bieten, sich $\mathrm{zu}$ vernetzen und neue Kontakte $\mathrm{zu}$ knüpfen. Deshalb wurden einerseits potenziell interessante (deutsche) Institutionen vor Ort u.a. für Gastvorträge hinzugezogen und andererseits mit der Thematik befasste KollegInnen von litauischen Hochschulen als GastreferentInnen eingeladen.

\subsection{Teilnehmergruppe und Heterogeni- tät}

An dem Kurs nahmen 8 Deutschlehrkräfte von 6 Hochschulen aus ganz Litauen teil. Dies ist insofern erfreulich, als es ein explizites Ziel war, mit dem Kurs auch kleinere Hochschulen in der Fläche zu erreichen.

Die Gruppe zeichnete sich in mehrfacher Hinsicht durch Heterogenität aus. Die Teilnehmerinnen kamen einerseits von Universitäten (Fachsprachenzentren und Germanistische Institute) und andererseits von Kollegien. Zudem waren nicht alle Teilnehmerinnen fachlich auf Wirtschaftsdeutsch spezialisiert; teils unterrichten sie in anderen Fachdeutsch-Segmenten, z.B. Studierende der Geistesoder der Naturwissenschaften, teils erteilen sie auch allgemeinen Unterricht in deutscher Sprache. Dies ist jedoch nicht 
als Widerspruch zur intendierten Zielgruppe zu sehen, da Hochschullehrkräften in Litauen generell sehr viel Flexibilität hinsichtlich der zu unterrichtenden Kurse abverlangt wird, d.h. die im Kurs vermittelten Kenntnisse und Fertigkeiten sind, abgesehen von den ohnehin auf anderen Sprachunterricht übertragbaren Momenten, eine gute Investition für eine mögliche zukünftige Tätigkeit. Eine große Herausforderung war zudem, dem Umstand gerecht $\mathrm{zu}$ werden, dass die Teilnehmerinnen ihren Unterricht auf sehr unterschiedlichen Sprachniveaus (A2/B1 bis C2) erteilen.

Um die Kursinhalte möglichst gut den Bedürfnissen und Erwartungen der Kursteilnehmerinnen anpassen zu können, wurde vor Kursbeginn ein Fragebogen zur Ermittlung besonderer Bedarfe und/oder Wünsche verschickt. Auf Basis dieser Ergebnisse wurde dann die konkrete inhaltliche Ausgestaltung des Kurses vorgenommen.

\subsection{Vorbereitung und Rahmenbedin- gungen}

Die organisatorische und inhaltliche Kursplanung von Kursorganisator und Gastdozentin war eng aufeinander abgestimmt und gestaltete sich dadurch zeitintensiv; sie scheint jedoch durch das Ergebnis gerechtfertigt.

Obwohl bereits bei der Beantragung des Kurses beim DAAD ein konkreter Plan und entsprechende Zusagen von kooperierenden Personen und Institutionen vorlagen, erforderte die Terminplanung während des Kurses viel Flexibilität, da mehrmals Veranstaltungen kurzfristig verschoben werden mussten. Dass dennoch alle geplanten Termine im Laufe des
Kurses zustande kamen, ist sehr erfreulich.

Aufgrund dienstlicher Verpflichtungen (Prüfungen, Projektteilnahme) konnten nicht immer alle Teilnehmerinnen an allen Kurstagen anwesend sein. Da dies jedoch bereits im Vorfeld des Kurses im Rahmen der Bedarfsermittlung geklärt worden war, wurden die Kurstage stärker modularisiert als ursprünglich geplant und auf diese Weise Inkohärenzen wie unnötige Wiederholungen weitestgehend vermieden.

Der Kurs konnte unter sehr günstigen Rahmenbedingungen durchgeführt werden. Für die gesamte Kurszeit wurde seitens der Pädagogischen Universität Vilnius ein moderner, sehr gut ausgestatteter Unterrichtsraum mit den gängigen Multimedia-Geräten zu Verfügung gestellt. Als der Arbeitsatmosphäre sehr zuträglich erwies sich, dass die gesamte Gruppe um einen runden Tisch sitzen konnte. Internetbasierte Unterrichtssequenzen fanden in einem Computerraum statt.

Zur Vorbereitung wurden an die Teilnehmerinnen in der Woche vor Kursbeginn vier Texte ${ }^{1}$ verschickt, die von den Teilnehmerinnen als Vorbereitung auf die Fortbildung gelesen werden sollten (die im Literaturverzeichnis angegebenen Texte dienten mir als Gastdozentin zur Vorbereitung der Fortbildung und als Material-Grundlage in der Fortbildung selbst). Für den Kurs wurde den Kursteilnehmerinnen ein Ordner mit Materialien zur Verfügung gestellt, der sehr großen Anklang fand. Die Materialien waren teilweise als Anregung, teilweise zum möglichen Einsatz in eigenen Wirtschaftsdeutsch-Kursen gedacht. Hierbei handelte es sich insbe-

1 Baumann (2000); Blei (2002); Brünner (2000: Kapitel 2.1 und 2.2); Lenske/SchöpperGrabe/Seitenfuß/Weber (1999). 
sondere um wissenschaftliche Texte, Handouts, Textsortenbeispiele sowie Beispiele für Übungen und Übungssequenzen. Hinzu kamen im Kursverlauf Materialien der GastreferentInnen und von den Firmenbesuchen (z. B. Handouts, Folien, Literaturlisten, Infobroschüren, Zeitschriften).

\section{Inhalt und Ablauf der Fortbildung}

\subsection{Kursinhalte}

In der ersten Woche des Fortbildungskurses stand die Vermittlung von Kenntnissen im Vordergrund, während in der zweiten Woche der Schwerpunkt auf der Anwendung der Kenntnisse und der Erstellung von Unterrichtssequenzen lag. Der Kurs umfasste mit 70 Unterrichtsstunden an 10 Tagen ein dichtes Programm; hinzu kamen Veranstaltungen des Rahmenprogramms.

\subsection{Kurswoche 1: Vermittlung von Kenntnissen}

$\mathrm{Zu}$ Kursbeginn fand anhand von Zeitungsartikeln und Internet-Nachrichten eine kurze, auf Beispielen aus dem alltäglichen Leben basierte Einführung in den Bereich Wirtschaft bzw. Geschäftsbzw. Unternehmenskommunikation statt. Auch Wirtschaftsdeutsch-Prüfungen (z.B. Prüfung Wirtschaftsdeutsch International PWD), die ein Ziel in Wirtschaftsdeutsch-Kursen sein können, wurden in der Einführungsphase exemplarisch vorgestellt, um den $\mathrm{Zu}$ sammenhang zwischen Geschäfts- bzw. Unternehmenskommunikation und Aufgaben in Wirtschaftsdeutsch-Prüfungen transparent $\mathrm{zu}$ machen. Dass sich Wirtschaftsdeutsch bzw. Wirtschaftskommunikation als schwer abzugrenzender, uneinheitlicher Bereich darstellt (vgl. u. a. Brünner 2000: 21), wurde im Gastvortrag »Was ist Wirtschaftsdeutsch«? zu Beginn des Kurses deutlich; in einem weiteren Gastvortrag wurden »Texte im Fachsprachenunterricht « beleuchtet. In dieser Anfangsphase vermittelte der Kurs zudem Grundlagen in BWL und VWL.

Die Kursteilnehmerinnen ihrerseits stellten die Rahmenbedingungen für Wirtschaftsdeutsch und die Situation (Curricula, Kurse) an ihren Hochschulen vor. Dabei wurden Bedarf, Möglichkeiten und Probleme der derzeitigen Situation von den Kursteilnehmerinnen rege diskutiert.

Da dem Fortbildungskurs ein dynamisches, pragmatisch-funktionales Kontextmodell sprachlicher Kommunikation als Kursansatz zugrunde liegt, sollte im Wirtschaftsdeutsch-Unterricht die Entwicklung »fachkommunikativer Kompetenz« (Baumann 2000: 158 f.) im Vordergrund stehen, d.h. die

»Fähigkeit der jeweiligen Lerner [...], Fachtexte als interkulturell, sozial, situativ und funktional bestimmte, sachlogisch gegliederte, semantisch strukturierte, linear-sequentiell sowie hierarchisch organisierte sprachliche Einheiten zu produzieren bzw. zu rezipieren « (Baumann 2000: 159).

Um fachkommunikative Kompetenz für ein bestimmtes berufliches Umfeld (hier: Wirtschaft) entwickeln zu können, sollten im Sinne der "Bedarfsorientierung" (Fluck 1998: 947) die typischen Situationen unternehmensinterner und -externer Geschäfts- und Unternehmenskommunikation und somit die einschlägigen schriftlichen und mündlichen Textsorten Gegenstand des Wirtschaftsdeutsch-Unterrichts sein, die sich bei Bedarfsanalysen unter Geschäftsleuten als die wichtigsten herausgestellt haben (vgl. z. B. Müntzel/Tiittula 1995, Huhta 1999; Minkkinen/Reuter 2001).

Im Fortbildungskurs ging es daher in Bezug auf schriftliche Textsorten einerseits um Geschäftskorrespondenz (Geschäftsbrief, E-Mail, Fax) von der Kon- 
taktaufnahme bis zur Mahnung und andererseits um Berichte und um Protokolle (vgl. Müntzel/Tiittula 1995). Diese Textsorten wurden im Hinblick auf Textbaupläne und die dazugehörigen sprachlichen Mittel (Morphosyntax, Terminologie) näher betrachtet und anhand von Beispielen sowie realitätsnahen Übungssequenzen illustriert (vgl. Müntzel/ Steuer 2002).

$\mathrm{Zu}$ den typischen mündlichen Kontaktsituationen in der Geschäfts- und Unternehmenskommunikation gehören - neben der Kontaktaufnahme und -pflege mit (neuen) Mitarbeitern und (neuen) Geschäftspartnern - geschäftliche Besprechungen, Verhandlungen unternehmensinterner und -externer Art sowie Präsentationen (Rodenbeck 2007: 41). Bei den mündlichen Textsorten wurden daher für den beruflichen Alltag wichtige monologische und dialogische Textsorten wie Unternehmens- und Produktpräsentationen sowie Verhandlungen, deren sprachliche Handlungsmuster sowie die dazugehörigen sprachlichen Mittel (Morphosyntax, Terminologie) genauer besprochen (vgl. Brünner 2000).

In einem weiteren Schritt wurden einschlägige Lehr- und Lernmaterialien für Wirtschaftsdeutsch präsentiert (vgl. z. B. Becker/Braunert/Schlenker 2004; Braunert 2006; Eismann 2008). Zwar gibt es viele Lehrwerke für Wirtschaftsdeutsch, die Zielgruppe sind jedoch Geschäftsleute und nicht Studierende, die i. d. R. weniger Fachkenntnisse und wenig bzw. keine Praxiserfahrung besitzen (vgl. Funk 1992: 6), so dass oftmals die Anpassung der Lehr- und Lernmaterialien für die eigene Zielgruppe vonnöten ist. Die Kenntnis von Lehrwerken und anderen Materialien ist somit notwendig für die kritische Auseinandersetzung mit denselben und die Voraussetzung für die gezielte Nutzung bzw. Modifi- zierung bestehender Wirtschaftsdeutsch-Angebote für die eigenen Zwecke.

In Verbindung mit den Lehrwerken, die auf den Gemeinsamen europäischen Referenzrahmen für Sprachen (GER) (vgl. Europarat 2001) ausgerichtet sind, wurden im Fortbildungskurs Anwendungsmöglichkeiten des GER speziell für Wirtschaftsdeutsch aufgezeigt sowie seine Möglichkeiten und Begrenzungen für den Wirtschaftsdeutsch-Unterricht diskutiert (vgl. Glaboniat/Müller/Rusch/ Schmitz/Wertenschlag, Lukas 2005, insbesondere das Gruppenprofil Deutsch für Angestellte in Unternehmen, sowie Hanken's CEFR Profile). Des Weiteren wurden zusammen mit dem Goethe-Institut in Vilnius externe Prüfungen für Deutsch als Fremdsprache und Wirtschaftsdeutsch präsentiert. Da die Prüfung Wirtschaftsdeutsch International (PWD) (vgl. Perlmann-Balme 2000) in Litauen derzeit noch nicht angeboten wird, bei den Wirtschaftsdeutsch unterrichtenden Institutionen aber Interesse an der Prüfung besteht, wurde die PWD ausführlich vorgestellt.

Während der Fortbildung lernten die Kursteilnehmerinnen auch Internetressourcen für Wirtschaftsdeutsch kennen. Hierbei stand die praktische Arbeit im Vordergrund, wobei die Kursteilnehmerinnen die Internetressourcen auch selbst ausprobierten und für eigene Unterrichtszwecke auf deren mögliche Verwendbarkeit hin testeten. Aufgrund der unterschiedlichen Vorkenntnisse bei der Arbeit am Computer erwies es sich bei der Vorstellung einzelner Internetressourcen als schwierig, eine für alle Teilnehmerinnen passende Präsentationsgeschwindigkeit zu finden.

Ein weiterer Punkt, der im Kurs angesprochen wurde, war die fachliche Übersetzung in der Wirtschaftskommunikation (vgl. z. B. Stolze 1999), was für die 
Kursteilnehmerinnen einen gelungenen Einblick in dieses für die unterrichtliche Praxis in Litauen relevante Thema darstellte.

\subsection{Kurswoche 2: Anwendung der Kenntnisse}

Während in der ersten Kurswoche der Schwerpunkt auf der Vermittlung der erforderlichen Informationen lag, stand in der zweiten Woche Eigenarbeit im Mittelpunkt, wobei die in der ersten Woche vermittelten Kenntnisse die Basis für die weitere Kursarbeit bildeten. Dabei überlegten die Kursteilnehmerinnen zuerst, wie ein Curriculum für Wirtschaftsdeutsch unter Berücksichtigung der Rahmenbedingungen an der jeweiligen Hochschule entwickelt werden kann, welche Ziele damit verfolgt werden und welche Leitprinzipien dem Curriculum zugrunde liegen (vgl. dazu z. B. Lenske/Schöpper-Grabe/Seitenfuß/ Weber 1999; Baumann 2000; Breckle/ Båsk/Rodenbeck 2007). Im nächsten Schritt ging es um die Planung einzelner Kurse, wobei der Schwerpunkt darauf lag, wie man Wirtschaftsdeutsch-Kurse und Unterrichtssequenzen aufbauen kann (vgl. z. B. Funk 1992: 7 ff.; Lenske/ Schöpper-Grabe/Seitenfuß/Weber 1999; Funk 2001), um die Ziele des Curriculums zu erreichen.

Aufgrund des begrenzten Zeitrahmens konnten Curriculumentwicklung und Kursplanung allerdings nur exemplarisch behandelt werden. In zukünftigen Fortbildungen dieser Art sollten jedoch der konkreten Entwicklung von Curricula sowie der konkreten Kursplanung durch die Teilnehmenden mehr Zeit eingeräumt werden; auch die Vorbereitungstexte $\mathrm{zu}$ diesem Themenbereich könnten dann konkreter in den Kurs mit einbezogen werden. Beim Übergang von der Theorie zur Praxis hätte durch die Gegenüberstellung der Literatur zu fach- kommunikativer Kompetenz (Baumann 2000; Blei 2002) und konkreter Beispiele aus der unterrichtlichen Praxis (Übungssequenzen) zudem ein stärkerer Bezug zwischen beiden hergestellt werden können, als dies im Kurs der Fall war.

Die praktische Arbeit der zweiten Fortbildungswoche mündete in die Entwicklung eigener, vollständiger Unterrichtssequenzen. Für die Erarbeitung der eigenen Unterrichtssequenzen, bei der Partnerarbeit ausdrücklich erwünscht war, legten die Kursteilnehmerinnen zunächst die Zielgruppe, das Sprachniveau und das Ziel der Unterrichtssequenz fest. Anschließend suchten sie geeignetes Material - entweder nutzten sie bestehende Angebote für Lehr- und Lernmaterial, die sie für die eigenen Zwecke modifizierten, oder nicht-didaktisiertes Material, aus dem sie die Unterrichtsmaterialien für die Übungssequenz selbst entwickelten. Unterschätzt wurde von der Gastdozentin bei der Kursplanung die Zeit, die die Themen- und Materialfindung in Anspruch nimmt; hier sollte mehr Zeit eingeplant bzw. die Teilnehmerinnen von Kursbeginn an zur frühzeitigen Themen- und Materialfindung ermuntert werden. Nach einer ersten Einarbeitungsphase wurden die Konzepte der Kursteilnehmerinnen konstruktiv diskutiert; am Ende des Kurses wurden die fertigen Unterrichtssequenzen im Kurs präsentiert und wohlwollend-kritisch kommentiert. An dieser Stelle ist die hohe Qualität der von den Kursteilnehmerinnen selbst erstellten Unterrichtsmaterialien und Unterrichtssequenzen hervorzuheben.

\subsection{Ergänzung des Kursprogramms}

Ergänzt wurde das Kursprogramm durch informative Besuche bei zwei Firmen mit deutscher Beteiligung (DnBNord und HypoVereinsbank) und einen 
Gastvortrag eines Vertreters der DeutschBaltischen Handelskammer zu Handelsbeziehungen zwischen Litauen und Deutschland. Hierbei erhielten die Kursteilnehmerinnen nicht nur Informationen aus der Praxis, sondern es wurde intensiv diskutiert, wo und wie viel Deutsch man im Berufs-/Wirtschaftsleben in Litauen braucht und welchen Nutzen Deutschkenntnisse in Litauen grundsätzlich haben. Deutschkenntnisse dienen nicht nur als Zusatzqualifikation; in einigen Branchen wie den Banken werden gerade auch AbsolventInnen philologischer Fächer wegen ihrer analytischen Fähigkeiten sowie ihrer Sprachkenntnisse gesucht. Diese wichtigen Informationen können die Kursteilnehmerinnen motivierend an ihre Studierenden weitergeben.

Dass der Besuch des Presse- und Kulturreferenten der Deutschen Botschaft Wilna ans Ende der Fortbildung verschoben werden musste, erwies sich als durchaus positiv, denn neben einem kurzen Vortrag zur Bedeutung des Deutschen in der internationalen Wirtschaftskommunikation ließ der Besuch Raum für die vielseitige und substanzielle Diskussion ausgewählter Kursergebnisse mit dem Botschaftsvertreter. Bei den Kursteilnehmerinnen stieß dabei die Wertschätzung ihres Engagements und ihrer Leistung seitens des Botschaftsvertreters auf ein sehr positives Echo.

\subsection{Rahmenprogramm}

Während des Kurses wurde ein Rahmenprogramm angeboten, darunter ein geselliger Abend zum gegenseitigen Ken- nenlernen und ein sehr gelungenes gemeinsames Abendessen gegen Ende des Kurses. Ein Treffen mit VertreterInnen des sehr aktiven IPP Vereins ${ }^{1}$ Litauen, das nur zum Teil dem Rahmenprogramm zuzurechnen ist, verfolgte den Zweck, den Bekanntheitsgrad des Internationalen Parlamentsstipendiums über die Schiene des studienbegleitenden Deutschunterrichts zu erhöhen, um mehr potenzielle Interessierte ansprechen $\mathrm{zu}$ können.

\subsection{Kursevaluation}

In der Woche nach Kursende wurde ein Evaluationsbogen an die Teilnehmerinnen verschickt. Bei sehr hohem Rücklauf erhielt der Kurs - insbesondere in Bezug auf Organisation, Kursinhalte, Material und GastreferentInnen - eine sehr gute bis gute Beurteilung durch die Teilnehmerinnen. Positiv beurteilt wurde auch die Veröffentlichung der Ergebnisse des Fortbildungskurses. Hervorgehoben wurde zudem die äußerst positive Atmosphäre im Kurs. Es herrschte nicht nur eine angenehme, offene Atmosphäre unter den am Kurs Beteiligten (Kursteilnehmerinnen, Gastdozentin, Organisator, GastreferentInnen), sondern auch sehr großes Interesse der Kursteilnehmerinnen an den Kursinhalten, insbesondere an Didaktik und Methodik (Curriculumentwicklung, Kursplanung, Aufbau von Übungssequenzen für Wirtschaftsdeutsch-Kurse), und ein sehr positives Arbeitsklima im Kurs. Die relativ kleine Kursgröße war dabei von Vorteil. Alle Teilnehmerinnen beurteilten ihre Kursteilnahme erfreulicherweise so, dass sie

1 IPP sind Internationale Parlamentspraktika, die vom Deutschen Bundestag und der Universität Humboldt zu Berlin organisiert werden und jungen Menschen die Möglichkeit bieten, durch ein Praktikum beim deutschen Bundestag das parlamentarische Regierungssystem der Bundesrepublik Deutschland kennenzulernen. Ziel des IPP Vereins Litauen ist, die Kontakte unter den ehemaligen BundestagspraktikantInnen aus Litauen zu pflegen. 
glauben, durch den Kurs in Zukunft Nutzen zu haben.

Darüber hinaus wurden wohlwollendkonstruktive, wertvolle Hinweise für ggf. künftige Kurse dieser Art gegeben. Hier ist vor allem zu nennen, dass nicht alle Kursinhalte (z. B. fachliche Übersetzung) für alle Teilnehmerinnen relevant waren, der Wunsch, die zur Vorbereitung verschickten Texte noch stärker und konkreter in den Kurs einzubinden, und das Problem, zwischen Vorlesungszeit, Prüfungszeit und Ferien einen geeigneten Kurstermin zu finden.

Aus Sicht des Kursorganisators und der Gastdozentin erwiesen sich insbesondere folgende Punkte als problematisch:

- Wie sich im Nachhinein herausstellte, konnten durch die genutzten Kanäle zur Ankündigung der Fortbildung nicht alle potenziell interessierten Personen erreicht werden. Daher sollte für künftige Fortbildungen dieser Art über eine Verbesserung der Reichweite (z. B. durch Informationsplakate und -faltblätter) nachgedacht werden.

- Problematisch gestaltete sich auch die Terminierung des Kurses, denn an vielen Hochschulen Litauens ist der gewählte Zeitraum Anfang Juni Prüfungszeit. Generell dürfte es jedoch schwierig bzw. unmöglich sein, einen für alle passenden Termin zu finden, da die Angehörigen der Zielgruppe neben ihrer hochschulischen Lehrtätigkeit eine Reihe weiterer Beschäftigungen ausüben (müssen).

- Wegen der wenigen und zögerlichen Anmeldungen wurde zeitweise überlegt, den Kurs abzusagen. Die Entscheidung, die Veranstaltung trotzdem durchzuführen, hat sich als richtig erwiesen. Dass bei der Bewerbung eine kurze Begründung vorgesehen war, um ggf. eine begründete Entscheidung über die Vergabe der Kursplätze treffen zu können, erwies sich jedoch als unnötig.

\subsection{Publikation der Ergebnisse}

Aufgrund der Qualität der im Kurs entstandenen Unterrichtssequenzen wurden die Kursergebnisse unter dem Titel Fachdeutsch in Beispielen - Vorschläge zu Wirtschaftsthemen im studienbegleitenden Unterricht im Verlag der Pädagogischen Universität Vilnius (Breckle/Geyer 2008) publiziert. Das 120-seitige Büchlein enthält sechs Übungssequenzen mit detaillierten Angaben zu Zielgruppe, Sprachniveau, Ziel der Übungen sowie mit Kommentaren und Erläuterungen. Für die Publikation stellte zudem der Botschaftsvertreter seine motivierenden Überlegungen zur Bedeutung des Deutschen in der Wirtschaftskommunikation zur Verfügung.

\section{Resümee und Perspektive}

Aufgrund der Erfahrungen lässt sich im Hinblick auf Fortbildungen dieser Art folgendes Resümee ziehen: In der Phase der Antragstellung beim DAAD würde aus Sicht von Kursorganisator und Gastdozentin ein Antrag mit einem weniger detaillierten Kursschema mehr Vorlauf erlauben; es sollte ausreichen, die geplanten Inhalte mit Stundenzahl zu nennen, denn die konkreten Terminabsprachen erfolgen in Litauen in der Regel sehr spät. Der Kurs hat gezeigt, dass ein intensives Programm für die Teilnehmerinnen zumutbar ist und dass auch ein kleiner finanzieller Eigenbeitrag von den Teilnehmerinnen geleistet werden kann. Es ist auch deutlich geworden, dass engagierte KollegInnen vielfältig eingebunden sind, was sich beispielsweise auf die Anwesenheit während des Kurses auswirken kann. Trotz der Bedarfserfragung im Vorfeld des Kurses und entsprechender Kursplanung zeigte die Kursauswertung, dass nicht alle Kursinhalte für alle Teilnehmerinnen gleichermaßen relevant waren; hier könnte eine stärkere Binnendifferenzierung während des 
Fortbildungskurses eine Möglichkeit darstellen, die Kursinhalte noch besser auf die Bedürfnisse der Teilnehmenden abzustimmen. Die Kursevaluation hat den Eindruck bestätigt, dass es schwierig bzw. unmöglich zu sein scheint, einen für alle Interessierten passenden Termin für einen solchen Fortbildungskurs zu finden.

Insgesamt beurteilen Kursorganisator und Gastdozentin den Fortbildungskurs Wirtschaftsdeutsch für Hochschullehrkräfte in Litauen als sehr gelungen, sowohl was die Arbeit im Kurs als auch was die Netzwerkbildung und Multiplikatoreffekte betrifft. Die abschließende Kursevaluation unterstützt diesen Eindruck.

Für eine mögliche Wiederholung eines Fortbildungskurses Wirtschaftsdeutsch im bestehenden Kursformat, für den als erweiterter Teilnehmerkreis nicht nur Hochschullehrkräfte aus Litauen, sondern auch aus Lettland und Estland denkbar wären, ließe sich auf Basis der Erfahrungen und der Kursevaluation eine mögliche Nachfolgeveranstaltung noch verbessern.

\section{Literatur}

Baumann, Klaus-Dieter: »Die Entwicklung eines integrativen Fachsprachenunterrichts - eine aktuelle Herausforderung der Angewandten Linguistik«. In: Baumann, Klaus-Dieter; Kalverkämper, Hartwig; Steinberg-Rahal, Kerstin (Hrsg.): Sprachen im Beruf. Stand - Probleme - Perspektiven (Forum für Fachsprachen-Forschung, 38). Tübingen: Narr, 2000, 149-173.

Becker, Norbert; Braunert, Jörg; Schlenker, Wolfram: Unternehmen Deutsch. Grundkurs. Stuttgart: Klett, 2004.

Blei, Dagmar: »Aufgaben zur Entwicklung einer fachkommunikativen Kompetenz«, Info DaF 29, 4 (2002), 289-305.

Bolten, Jürgen: »Fremdsprache Wirtschaftsdeutsch: Bestandsaufnahme und Perspektiven«. In: Müller, Bernd-Dietrich (Hrsg.): Interkulturelle Wirtschaftskommu- nikation (Studium Deutsch als Fremdsprache - Sprachdidaktik, 9). 2. Auflage. München: iudicium, 1993, 71-91.

Braunert, Jörg: Unternehmen Deutsch. Aufbaukurs. Stuttgart: Klett, 2006.

Breckle, Margit; Båsk, Märta; Rodenbeck, Rolf: Wirtschaftssprache Deutsch in Studium und Beruf. Curriculumentwicklung an der Schwedischen Wirtschaftsuniversität in Finnland. Helsinki: Schwedische Wirtschaftsuniversität, 2007 (Forschungsberichte, 63). Online: http://openax.shh.fi:8180/dspace/ bitstream/10227/256/1/63-978-951-555957-9.pdf

Breckle, Margit; Geyer, Klaus (Hrsg.): Fachdeutsch in Beispielen - Vorschläge zu Wirtschaftsthemen im studienbegleitenden Unterricht. Vilnius: Verlag der Pädagogischen Universität Vilnius, 2008.

Brünner, Gisela: Wirtschaftskommunikation. Linguistische Analyse ihrer mündlichen Formen (Reihe Germanistische Linguistik, 213). Tübingen: Niemeyer, 2000.

Buhlmann, Rosemarie; Fearns, Anneliese: Einführung in die Fachsprache der Betriebswirtschaft. Band I-II. München: GoetheInstitut, 1989.

Buhlmann, Rosemarie; Fearns, Anneliese: Einführung in die Fachsprache der Betriebswirtschaft. Band III. München: GoetheInstitut, 1995.

Eismann, Volker: Wirtschaftskommunikation Deutsch. Berlin; München: Langenscheidt, 2008.

Europarat (Hrsg.): Gemeinsamer europäischer Referenzrahmen für Sprachen: lernen, lehren, beurteilen (in Zusammenarbeit mit Sheils, Joseph. Übersetzung: Jürgen Quetz in Zusammenarbeit mit Raimund Schieß und Ulrike Sköries; Übersetzung der Skalen: Günther Schneider). Berlin; München: Langenscheidt, 2001.

Fluck, Hans-Rüdiger: »Bedarf, Ziele und Gegenstände fachsprachlicher Ausbildung «. In: Hoffmann, Lothar; Kalverkämper, Hartwig; Wiegand, Herbert Ernst (Hrsg.): Fachsprachen. Ein internationales Handbuch zur Fachsprachenforschung und Terminologiewissenschaft (Handbücher zur Sprach- und Kommunikationswissenschaft, 14.1). 1. Halbband. Berlin; New York: de Gruyter, 1998, 944-954.

Funk, Hermann: »Berufsbezogener Deutschunterricht. Grundlagen - Lern- 
ziele - Aufgaben «, Fremdsprache Deutsch Sondernummer 1992, 4-16.

Funk, Hermann: »Berufsbezogener Deutschunterricht - Deutsch als Fremdund Zweitsprache für den Beruf «. In: Helbig, Gerhard; Götze, Lutz; Henrici, Gerd; Krumm, Hans-Jürgen (Hrsg.): Deutsch als Fremdsprache: ein internationales Handbuch. 2. Halbband (Handbücher zur Sprach- und Kommunikationswissenschaft, 19.2). Berlin; New York: de Gruyter, 2001, 962-973.

Glaboniat, Manuela; Müller, Martin; Rusch, Paul; Schmitz, Helen; Wertenschlag, Lukas: Profile Deutsch. Lernzielbestimmungen. Kannbeschreibungen. Kommunikative Mittel. Niveau A1-A2, B1-B2, C1-C2 (mit CDRom). Berlin; München: Langenscheidt, 2005.

»Hanken's CEFR Profile«. In: Breckle, Margit; Båsk, Märta; Rodenbeck, Rolf: Wirtschaftssprache Deutsch in Studium und Beruf. Curriculumentwicklung an der Schwedischen Wirtschaftsuniversität in Finnland (Forschungsberichte, 63). Helsinki: Schwedische Wirtschaftsuniversität, 2007, 86-92. Online: http://openax.shh.fi:8180/d space/bitstream/10227/256/1/63-978-951-555-9579.pdf

Huhta, Marjatta: Language/Communication Skills in Industry and Business - Report for Prolang/Finland. Helsinki: National Board of Education, 1999. Online: http:// www.edu.fi/julkaisut/skills42.pdf

Hundt, Markus: „Neuere institutionelle und wissenschaftliche Wirtschaftsfachsprachen «. In: Hoffmann, Lothar; Kalverkämper, Hartwig; Wiegand, Herbert Ernst (Hrsg.): Fachsprachen. Ein internationales Handbuch zur Fachsprachenforschung und Terminologiewissenschaft. 2. Halbband (HSK, 14.2). Berlin; New York: de Gruyter, 1998, 1296-1304.

Lenske, Werner; Schöpper-Grabe, Sigrid; Seitenfuß, Gabriele; Weber, Bärbel: Entwicklung von Curricula für den berufsbezogenen Fremdsprachenunterricht (Beiträge zur Gesellschafts- und Bildungspolitik. Institut der deutschen Wirtschaft). Köln: Deutscher Instituts-Verlag, 1999.

Minkkinen, Eila; Reuter, Ewald: FinnischDeutsche Unternehmenskommunikation in der Region Pirkanmaa. Zwischenbericht. Tampere: Universität Tampere, 2001.

Müntzel, Uta; Steuer, Outi: Perfekte Geschäftskommunikation per Brief, Fax und E-Mail.Ein netzgestütztes Lern- und Trainingsprogramm. Helsinki: WSOY, 2002. Online: http:// opit.wsoy.fi/LMS_newlogin.asp (mit persönlichem Passwort zugänglich).

Müntzel, Uta; Tiittula, Liisa: Deutsch im finnisch-deutschen Handel. Eine Bedarfsanalyse (Helsingin kauppakorkeakoulun julkaisuja D-215). Helsinki: Helsingin kauppakorkeakoulu, 1995.

Perlmann-Balme, Michaela: Prüfung Wirtschaftsdeutsch International. Handbuch. Prüfungsziele. Testbeschreibung. München: Goethe-Institut, 2000.

Reuter, Ewald: »Wirtschaftstexte«. In: Helbig, Gerhard; Götze, Lutz; Henrici, Gerd; Krumm, Hans-Jürgen (Hrsg.): Deutsch als Fremdsprache: ein internationales Handbuch. 1. Halbband (HSK, 19.1). Berlin; New York: de Gruyter, 2001, 573-582.

Rodenbeck, Rolf: »Mündliche Geschäftskommunikation: Unternehmenspräsentation und Verhandlung «. In: Breckle, Margit; Båsk, Märta; Rodenbeck, Rolf: Wirtschaftssprache Deutsch in Studium und Beruf. Curriculumentwicklung an der Schwedischen Wirtschaftsuniversität in Finnland (Forschungsberichte, 63). Helsinki: Schwedische Wirtschaftsuniversität, 2007, 40-49. Online: http://openax.shh.fi:8180/dspace/ bitstream/10227/256/1/63-978-951-555957-9.pdf

Stolze, Radegundis: Die Fachübersetzung. Eine Einführung. Tübingen: Narr, 1999.

\section{Margit Breckle}

Studium der Fächer Deutsch als Fremdsprache, Anglistik und Wirtschaftswissenschaften an den Universitäten Bielefeld und Vaasa (Finnland); 1997 M. A. in Bielefeld; 1998-2002 Lehre (DaF) an verschiedenen Universitäten in Skandinavien; 2004 Promotion an der Universität Göteborg (Schweden); 2002-2007 DAADLektorin an der Schwedischen Wirtschaftsuniversität in Helsinki (Finnland); seit 2007 an der Hochschule Konstanz. 


\section{Anhang:}

Fragebogen zur Ermittlung besonderer Bedarfe und/oder Wünsche:

\section{Wirtschaftsdeutsch}

für Hochschullehrkräfte in Litauen

vom 4. bis 15. Juni 2007, Pädagogische Universität Vilnius

Um die Fortbildungsveranstaltung möglichst genau auf Ihre Wünsche und Bedürfnisse abstimmen zu können, möchten wir Sie über Ihre Vorerfahrungen im Bereich Wirtschaftsdeutsch befragen.

\begin{tabular}{|l|l|l||}
\hline Akad. Grad & Name & Vorname \\
\hline Institution & \\
\hline
\end{tabular}

Haben Sie Erfahrungen im Unterricht von Wirtschaftsdeutsch?

Bitte auswählen: ja / ein bisschen / nein

Was halten Sie bei der Vermittlung von Wirtschaftsdeutsch für wichtig (Schwerpunkte)?

Welche Wirtschaftsdeutsch-Kurse unterrichten Sie?

\begin{tabular}{|l|l|l|l|l||}
\hline Kurstitel & Inhalte/Themen & Zielgruppe & Stundenzahl & Sprachniveau \\
\hline & & & & $\begin{array}{l}\text { Bitte auswählen: } \\
\text { A1/A2/B1/B2/C1/C2 }\end{array}$ \\
\hline & & & & $\begin{array}{l}\text { Bitte auswählen: } \\
\text { A1/A2/B1/B2/C1/C2 }\end{array}$ \\
\hline & & & & $\begin{array}{l}\text { Bitte auswählen: } \\
\text { A1/A2/B1/B2/C1/C2 }\end{array}$ \\
\hline
\end{tabular}

Welche Unterrichtsmaterialien benutzen Sie für Ihre Kurse (z. B. Lehrwerke, selbst erstellte Materialien, ...)?

Haben Sie Erfahrung in der Erstellung von Unterrichtsmaterialien für WirtschaftsdeutschKurse (vollständig selbst erstellt oder auf Basis von vorhandenen Materialien, z. B. Lehrwerken)?

Bitte auswählen: ja / ein bisschen / nein

Wenn ja (oder ein bisschen), bitte genauer beschreiben:

Haben Sie Erfahrung in der Planung vollständiger Wirtschaftsdeutsch-Kurse?

Bitte auswählen: ja / ein bisschen / nein

Wenn ja (oder ein bisschen), bitte genauer beschreiben:

Haben Sie Erfahrung in der kursübergreifenden Curriculumentwicklung für Wirtschaftsdeutsch?

Bitte auswählen: ja / ein bisschen / nein

Wenn ja (oder ein bisschen), bitte genauer beschreiben:

Welche Erwartungen und Wünsche haben Sie an die Fortbildung? 